\title{
REVISIÓN
}

REVIEW

\section{ESTRATEGIAS PROFILÁCTICAS PARA DISFAGIA OROFARÍNGEA EN PACIENTES CON CÁNCER DE CABEZA Y CUELLO: REVISIÓN NARRATIVA}

\author{
PROPHYLACTIC STRATEGIES FOR OROPHARYNGEAL DYSPHAGIA IN PA- \\ TIENTS WITH HEAD AND NECK CANCER: A NARRATIVE REVIEW
}

\begin{abstract}
Camilo Morales ${ }^{1}$, Joaquín Gay², Tomás Esparza
\section{RESUMEN}

El cáncer de cabeza y cuello es una patología prevalente a nivel mundial. Su abordaje consiste, en buena parte de los casos, de quimiorradioterapia, la cual, dentro de sus múltiples consecuencias, comprometelaanatomíay elfuncionamiento de órganosrelacionadas a la deglución, generando disfagia orofaríngea. En ese sentido, el manejo fonoaudiológico predominantemente se lleva a cabo con ejercicios implementados posterior a la manifestación de la disfagia, con creciente interés sobre el manejo profiláctico del cuadro, existiendo variados estudios sobre la utilidad de ejercicios y maniobras relacionadas. En consecuencia, el objetivo de la presente revisión es presentar recursos terapéuticos basados en evidencia de calidad para el manejo proactivo de esta alteración, orientando la decisión clínica sobre su uso.
\end{abstract}

\section{ABSTRACT}

Head and neck cancer is a worldwide prevalent pathology. Its approach consists, in most cases, on chemoradiotherapy. Within its multiple consequences, compromises anatomy and functioning of structures related to swallowing, generating oropharyngeal dysphagia. In this context, Speech and Language Pathologist management is predominantly carried out with exercises implemented after the manifestation of dysphagia, existing increasing interest in prophylactic management of the condition, with several studies on the usefulness of exercises and related available maneuvers. Consequently, the objective of the present review is to present therapeutic resources based on good quality evidence for the proactive management of this alteration, guiding the clinical decision on its use.

\section{INTRODUCCIÓN}

El cáncer de cabeza y cuello (CCC) representa

Eun amplio concepto, abarca las neoplasias malignas epiteliales de los tractos gastrointestinales y de las vías respiratorias superiores, incluyendo gran cantidad de subsitios, tales como la cavidad nasal, senos paranasales, nasofaringe, cavidad oral, orofaringe, laringe, hipofaringe y glándulas salivales. Constituye la quinta neoplasia maligna más común en todo el mundo, comprende el $6 \%$ de estas, con al menos 500,000 casos a nivel mundial'. En su gran mayoría, las neoplasias epiteliales mencionadas son carcinomas de células escamosas, cuyos factores de riesgo más importantes son el consumo de alcohol, tabaco, infecciones virales, exposición ocupacional, radiación, factores dietéticos y susceptibilidad genética ${ }^{2,3}$.
El tratamiento para el CCC es complejo, dependiendo no sólo de la localización de la enfermedad, sino también de la intrincada anatomía, con estructuras sanas y tumorales que comparten espacios muy reducidos, considerando siempre la importancia de preservar la función del órgano afectado. Si bien la radioterapia (RT) y la cirugía siguen siendo las dos opciones principales de tratamiento, la terapia sistémica (quimioterapia y terapia dirigida) se ha convertido recientemente en una parte integral del tratamiento multidisciplinario. La elección de la modalidad depende de los factores del paciente, el sitio primario, el estadio clínico y la resecabilidad del tumor 4 .

Aproximadamente del $30 \%$ al $40 \%$ de los pacientes presentan enfermedad temprana (estadios I y II). Estos pacientes pueden ser tratados
Magíster en Epidemiología Clínica. Carrera de Fonoaudiología. Departamento de Procesos Terapéuticos. Facultad de Ciencias de la Salud. Universidad Católica de Temuco, Chile 2 Magíster en Neurociencias. Universidad Autónoma de Chile, Facultad de Ciencias de la Salud, Carrera de Fonoaudiología. Temuco, Chile

${ }^{3}$ Licenciado en Fonoaudiología. Servicio de Medicina Física y Rehabilitación, Hospital Base de Osorno, Chile.

Correspondencia a: Camilo Morales Cárdenas Correo electrónico: joaquin.gay@uautonoma.cl Telefono: +(56) (2) 23036000

Palabras clave: Neoplasias de cabeza y cuello; Trastornos de Deglución; Terapéutica

Keywords: Head and neck neoplasms; Deglutition disorders; Therapeutics

Procedencia y arbitraje: no comisionado, sometido a arbitraje externo.

Recibido para publicación: 20 de abril 2020 Aceptado para publicación: 20 de junio 2020

Citar como:

Morales C, Gay J, Esparza T. Estrategias profilácticas para disfagia orofaríngea en pacientes con cáncer de cabeza y cuello: revisión narrativa Rev Cient Cienc Med 2020; 23(1): 69-76 
eficazmente con cirugía o RT definitiva. Ambas modalidades dan como resultado tasas similares de control y supervivencia locorregional, por lo que la elección generalmente se basa en la morbilidad y el resultado funcional que se puede esperar ${ }^{5}$.

Sin embargo, más de la mitad de los pacientes presentan enfermedad locorregional avanzada (estadio III o IV) en el momento del diagnóstico. El manejo de estos requiere medidas agresivas y concertadas, y sigue siendo un desafío clínico. Los pacientes pueden ser tratados con escisión quirúrgica completa, seguida de RT postoperatoria o primaria (con o sin quimioterapia). Hasta hace poco, se informaba que las tasas de supervivencia a 5 años estaban por debajo del 30\% para pacientes con enfermedad en estadio IV y del $40 \%$ para todos los tumores avanzados locorregionalmente, en cambio, la intensificación del tratamiento de RT utilizando horarios de fraccionamiento alterados y quimioterapia concomitante, ha dado como resultado un control locorregional y tasas de supervivencia significativamente mejoradas ${ }^{6}$. No obstante, dichas terapias utilizadas de manera paralela, se asocian con altas tasas de complicaciones, entre ellas, problemas de deglución temprana y tardía, secundario a alteraciones en tejidos blandos y neuronales ${ }^{4,7,8}$.

Disfagia como consecuencia de radio-quimioterapia.

La disfagia, como alteración para deglutir líquidos, sólidos y medicamentos durante la etapa orofaríngea y/o esofágica de la deglución, comúnmente derivada de los tratamientos previamente descritos, es una complicación de índole multifactorial y con alto riesgo de mortalidad. Se reporta una prevalencia aproximada de un $30-50 \%$, en pacientes sometidos a tratamientos intensivos no quirúrgicos, como RT, incrementando las alteraciones deglutorias y uso de gastrostomía endoscópica percutánea (GEP) al recibir terapias concomitantes como quimioterapia ${ }^{8}$.

Otros factores asociados a una mayor incidencia y severidad son la edad, el estado nutricional, el estadio tumoral, la localización de la irradiación (con mayor riesgo en bilateral) y los tumores en zona faríngea?

La alteración de la deglución puede ser temprana (posterior a quimio o radioterapia), e incluso tardía, es decir, posterior a un año del tratamiento ${ }^{8,10,11}$.Dentro de las estructuras más afectadas en el tratamiento para CCC, se reportan los constrictores faríngeos (principalmente faríngeo superior), el esfínter esofágico superior, la epiglotis, las secciones glótica y supraglótica de la laringe, y la base de la lengua ${ }^{3,4}$. Estas estructuras, transcurridos los primeros meses posterior a la quimioradioterapia (QR), se vuelven edematosas y con el tiempo algunos tejidos se fibrosan, constituyéndose en una lesión crónica, que impacta en la coordinación de movimientos deglutorios, asi mismo en la contracción y en la elevación laríngea.

Existen casos de disfagia tardía que resultan de mono o polineuropatías de nervios craneales, derivados de la RT, que también contribuyen al trastorno deglutorio, lo que podría añadir disminución de sensación laríngea y alteración del peristaltismo ${ }^{3}$. A lo anterior, se suma la atrofia por desuso, que estimula una remodelación adversa de los músculos del tracto aerodigestivo $^{12}$, incrementando el riesgo de aspiración sintomática y silente de los alimentos, en este último grupo ${ }^{13}$.

Otra de las funciones afectadas tiene relación con la disminución de fuerza espiratoria (PEmax y PeakFlow Tos) en pacientes con CCC posradiación, lo que sugiere que las alteraciones de la protección de las vías respiratorias pueden extenderse más allá de los mecanismos laringofaríngeos alterados en el campo de tratamiento local ${ }^{14}$.Todas estas alteraciones conllevan un impacto en el proceso deglutorio general.

La disminución en la retracción de la base de la lengua y constrictores faríngeos, resulta en un declive de la propulsión del bolo alimenticio hacia la faringe y una disminución de la elevación laríngea al deglutir, paralelo a una reducción del movimiento de basculación de la epíglotis, generando como consecuencia, un riesgo elevado de residuos en valléculas y senos piriformes o falsa vía después de la deglución, lo cual evidencia una relajación incompleta del esfínter cricofaríngeo ${ }^{15,16}$.

Existe además sequedad de la boca (xerostomía) por disfunción parotídea, como una de las complicaciones más frecuentes en estos tratamientos, impactando en la cantidad, volumen, consistencia (espesa) y pH de la saliva secretada, lo cual dificulta la formación y homogenización del bolo alimenticio, aumentando el riesgo de penetración o aspiración del alimento. Finalmente, la mucositis provoca faringoalgia y 
odinofagia ${ }^{17}$. Ambas complicaciones dificultan la deglución y también el habla, y aumentan el riesgo de sufrir infecciones orales o caries dentales ${ }^{18,19}$. La disfagia, indudablemente, tiene claras consecuencias negativas en la calidad de vida de las personas con CCC, dificultando el disfrute del proceso de alimentación, contemplando tiempos de alimentación más largos, restricción en la ingesta de algunos tipos de alimentos, miedo hacia la alimentación (por penetración o aspiración laríngea) o incapacidad de comer en público, resultando en aislamiento social y, en algunos casos, depresión ${ }^{20}$, situación que se complejiza aún más cuando estos usuarios se ven obligados a optar por una vía alternativa de alimentación.

La terapia fonoaudiológica, en ese sentido, se erige como una opción importante para los usuarios con CCC. A través de ejercicios y maniobras, permiten abordar y mejorar aspectos del proceso deglutorio, buscando mejorar la calidad de vida de las personas. Los ejercicios de deglución dirigidos a estas alteraciones específicas se han dispuesto con el objetivo de compensar, optimizar o mejorar la movilidad y motilidad de las estructuras antes descritas. Sin embargo, su utilización se ha llevado a cabo predominantemente después de completar el tratamiento para CCC. Al respecto, se reportan resultados favorables, específicamente en el rango de movimiento mandibular, de la lengua al contacto con la pared posterior de la faringe, la elevación laríngea y la contracción faríngea, lo que resulta en la reanudación de la alimentación oral y la interrupción de la alimentación alternativa, a través de sonda nasogástrica, gastrostomía u otras opciones acordes a la realidad particular de los usuarios ${ }^{21-24}$.

Con las mejoras en la función deglutoria, ha aumentado el interés en el uso de ejercicios de deglución profilácticos para prevenir o minimizar la disfunción de deglución posterior a la QR. De hecho, algunos centros de tratamiento del cáncer recomiendan ejercicios de deglución profilácticos para todos sus pacientes sometidos a $\mathrm{QR}^{25,26}$. La terapia de deglución proactiva, respaldada por estudios observacionales y ensayos aleatorios, se ha convertido en atención de rutina en variadas instituciones ${ }^{21,27-30}$. Sin embargo, desde un punto de vista fonoaudiológico, no se encuentran sistematizados los ejercicios o maniobras que han resultado de utilidad en estudios de mayor nivel de evidencia. Consi- derando lo anterior, es necesario revisar los datos disponibles para realizar recomendaciones y medidas de apoyo terapéutico profiláctico, así como entregar lineamientos para futuras investigaciones.

METOdOLOGÍA DE BÚSQUEDA DE INFORMACIÓN

Se realizó una búsqueda en la base de datosPubmed, Google Académico y Sciencedirect, utilizando los siguientes términos y códigosbooleanos: ("head and neck cancer" OR "HNC" AND "chemoradiation" OR "radiotherapy" "chemoradiotherapy" AND "swallowing disorders" OR dysphagia not stroke) AND ("prophylactic" OR pretreatment AND maneuvers OR exercises OR "swallowing exercises" OR program) AND (effectiveness OR "QOL" OR "swallowing function" OR "FOIS" OR "PAS" OR "mouth opening" OR xerostomia OR trismus OR aspiration OR "feeding tube" OR videofluoroscopy OR "FEES"). La búsqueda arrojó 61 resultados, seleccionando 9 de ellos basados en título, abstract, relevancia del tema y antigüedad (menor a 10 años). Se utilizó el filtro metodológico "ensayos clínicos aleatorizados", por considerarse el máximo nivel de evidencia para estudios primarios. Finalmente, del total de artículos seleccionados, se escogieron 6 por criterio de inclusión, es decir, estudios que abordan exclusivamente el manejo profiláctico de la disfagia en usuarios con CCC. Por otra parte, se efectuó una segunda revisión de tipo clásico según lo propuesto Montero et al. ${ }^{31}$, en donde se utilizan artículos extraídos de otros motores de búsqueda y bibliografía pertinente para complementar lo encontrado en la búsqueda principal. Se reportaron aquellas maniobras o ejercicios que probaron ser efectivas en estudios clínicos aleatorizados y controlados.

\section{DESARROLLO}

Los resultados expresan cuáles son los ejercicios deglutorios utilizados y recomendados con fin profiláctico en usuarios con CCC y riesgo de presentar disfagia por RT o QR, además de cuál sería la intensidad y duración de la terapia con base en resultados obtenidos en estudios de ensayos clínicos revisados. En ese contexto, la Tabla 1 provee una descripción detallada de cada estudio, los recursos utilizados y el reporte de los principales cambios en los usuarios que 
Tabla 1. Detalle de estudios y recursos terapéuticos seleccionados.

\begin{tabular}{|c|c|c|c|c|c|}
\hline & Estudio & de inter- & & citación & \\
\hline $\begin{array}{lr}\text { Van } & \text { der } \\
\text { molen } & \text { et } \\
\text { al.(38) } & \end{array}$ & $\begin{array}{l}\text { E n s a y o } \\
\text { clínico ale- } \\
\text { atorizado, } \\
\text { controlado. }\end{array}$ & $\begin{array}{l}\text { Intervención } \\
\text { profiláctica } \\
\text { experimen- } \\
\text { tal } \quad(\mathbf{n}=\mathbf{2 4})\end{array}$ & $\begin{array}{l}\text { - Ejercicios pasivos de apertura } \\
\text { oral lenta, con aparato Thera- } \\
\text { bite Jaw Motion Rehabilitation } \\
\text { System. } \\
\text {-Deglución con esfuerzo. } \\
\text {-Maniobra de Masako. } \\
\text {-Deglución super-supraglótica. } \\
\text {-Ejercicios de fortalecimiento: } \\
\text { deglución con la lengua eleva- } \\
\text { da hacia el paladar, mantenien- } \\
\text { do la apertura oral a un } 50 \% \text { de } \\
\text { su máximo. } \\
\text {-Ejercicios de rango de movi- } \\
\text { miento mandibular. }\end{array}$ & $\begin{array}{l}10 \text { reps. } 3 \text { veces al día. } \\
\text { Con Therabite. } \\
\text { 5reps. } \\
3 \text { veces al día. para el resto } \\
\text { de maniobras/ ejercicios. }\end{array}$ & $\begin{array}{l}\text { - Mayor apertura oral. } \\
\text { - Disminución en puntuación FOIS } \\
\text { (funcionalidad deglutoria). } \\
\text { - Menor cantidad de resid- } \\
\text { uos faríngeos a la VFC. }\end{array}$ \\
\hline $\begin{array}{l}\text { C a r n a - } \\
\text { by-Mann et } \\
\text { al. }(27)\end{array}$ & $\begin{array}{l}\text { E n s a y o } \\
\text { clínico ale- } \\
\text { atorizado, } \\
\text { controlado. }\end{array}$ & $\begin{array}{l}\text { Tratamien- } \\
\text { to de alta } \\
\text { intensidad, } \\
\text { profilácti- } \\
\text { co } \quad(\mathbf{n}=\mathbf{2 0})\end{array}$ & $\begin{array}{l}\text { miento manqioular. } \\
\text { - Ejercicios de falsetto. } \\
\text {-Presión con la lengua contra } \\
\text { resistencia. } \\
\text {-Deglución con esfuerzo. } \\
\text {-Ejercicios de movimiento } \\
\text { mandibular contra resistencia } \\
\text { (Therabite). }\end{array}$ & $\begin{array}{l}4 \text { series de } 10 \text { reps., } 2 \text { vec- } \\
\text { es al día. cada serie de } 10 \\
\text { minutos de duración. }\end{array}$ & $\begin{array}{l}\text { - Menor deterioro de la musculatura } \\
\text { deglutoria (geniogloso, hiogloso, mi- } \\
\text { lohioídea) durante la QR. } \\
\text { - Menor severidad de disfagia (al } \\
\text { evaluar con MASA). } \\
\text { - Disminución en puntuación FOIS. } \\
\text { - Menor descenso en rango de aper- } \\
\text { tura bucal, agudez quimio sensorial y } \\
\text { salivación. }\end{array}$ \\
\hline $\begin{array}{l}\text { Kotz } \\
\text { al.(29) }\end{array}$ & $\begin{array}{l}\text { E n s a y o } \\
\text { clínico ale- } \\
\text { atorizado, } \\
\text { controlado. }\end{array}$ & $\begin{array}{l}\text { Intervención } \\
\text { profilácti- } \\
\text { ca } \quad(\mathbf{n}=\mathbf{1 0})\end{array}$ & $\begin{array}{l}\text { - Maniobra de Deglución con } \\
\text { esfuerzo (con agua o en seco). } \\
\text {-Maniobra de Masa- } \\
\text { ko (con agua o en seco). } \\
\text { - Ejercicio de retracción lingual. } \\
\text {-Maniobra de Mendelsohn (con } \\
\text { saliva). }\end{array}$ & $\begin{array}{l}3 \text { series de } 10 \text { reps, } 3 \text { vec- } \\
\text { es al día, diariamente. }\end{array}$ & $\begin{array}{l}\text { - Disminución en puntuación FOIS. } \\
\text { Calidad de vida: } \\
\text { - Normalización deladietaporvía oral. } \\
\text { - Capacidad de comer en público. }\end{array}$ \\
\hline $\begin{array}{l}\text { Van der } \\
\text { Mollen et } \\
\text { al. (33) }\end{array}$ & $\begin{array}{l}\text { E n s a y o } \\
\text { clínico ale- } \\
\text { atorizado, } \\
\text { controlado. }\end{array}$ & $\begin{array}{l}\text { Intervención } \\
\text { profilácti - } \\
\text { ca } \quad(\mathbf{n}=\mathbf{1 5}) \text {. }\end{array}$ & $\begin{array}{l}\text {-Ejercicios pasivos de apertura } \\
\text { oral lenta, con aparato Thera- } \\
\text { bite. } \\
\text {-Ejercicios de fortalecimiento: } \\
\text { deglución con la lengua eleva- } \\
\text { da hacia el paladar, mantenien- } \\
\text { do la apertura oral a un } 50 \% \text { de } \\
\text { su máximo. }\end{array}$ & 10 reps. (3 veces al día). & $\begin{array}{l}\text { - Disminución en puntuación FOIS. } \\
\text { - Disminución residual sobre y bajo } \\
\text { el hioides (VFC). } \\
\text { - Disminución de episodios de xero- } \\
\text { stomía. } \\
\text { Calidad de vida: } \\
\text { - Normalización de la dieta por vía } \\
\text { oral (disminución en el uso de sonda } \\
\text { nasogástrica). }\end{array}$ \\
\hline $\begin{array}{l}\text { Mortensen } \\
\text { et al.(36) }\end{array}$ & $\begin{array}{l}\text { E n s a y o } \\
\text { clínico ale- } \\
\text { atorizado, } \\
\text { controlado. }\end{array}$ & $\begin{array}{l}\text { Intervención } \\
\text { profilácti- } \\
\text { ca } \quad(\mathbf{n}=\mathbf{1 9})\end{array}$ & 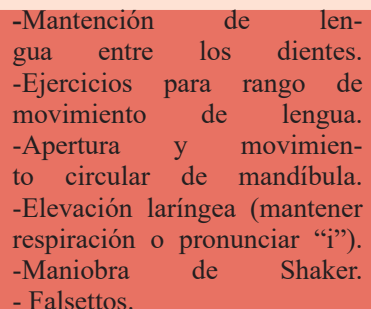 & $\begin{array}{l}7 \text { días a la semana, } \\
\text { de } 10 \text { a } 15 \text { minutos. } \\
\text { Todos los ejercicios, } 10 \\
\text { reps. (Salvo maniobra } \\
\text { de Shaker, que tiene su } \\
\text { propio protocolo, descrito } \\
\text { en la literatura). }\end{array}$ & $\begin{array}{l}\text { - Mejor función deglutoria a largo } \\
\text { plazo (después de } 1 \text { año de inter- } \\
\text { vención). }\end{array}$ \\
\hline $\begin{array}{l}\text { Messing et } \\
\text { al. (35) }\end{array}$ & $\begin{array}{l}\text { E n s a y o } \\
\text { clínico ale- } \\
\text { atorizado, } \\
\text { controlado. }\end{array}$ & $\begin{array}{l}\text { Intervención } \\
\text { profilácti - } \\
\text { ca } \quad(\mathbf{n}=\mathbf{2 9}) \text {. }\end{array}$ & $\begin{array}{l}\text {-Ejercicios de elongación / au- } \\
\text { mento del rango de movimiento } \\
\text { mandibular y de cuello (inclu- } \\
\text { ido uso de aparato Therabite). } \\
\text { - Ejercicios de elon- } \\
\text { gación/ aumento del rango } \\
\text { de movimiento de labios. } \\
\text {-Ejercicios de elongación y au- } \\
\text { mento de fuerza lingual. } \\
\text {-Maniobra de Masako. } \\
\text {-Maniobra de Mendelsohn. } \\
\text {-Deglución con esfuerzo. }\end{array}$ & $\begin{array}{l}\text { Ejercicios ejecutados de } \\
\text { manera diaria (7 días a } \\
\text { la semana) durante la } \\
\text { RQT (a excepción de la } \\
\text { semana de descanso del } \\
\text { tratamiento radio-quim- } \\
\text { ioterapia, en semana 4) } \\
\text { hasta } 3 \text { meses después de } \\
\text { la radio-quimioterapia. } \\
\text { - Protocolo } 7-7-7 \text { para } \\
\text { ejercicios de estira- } \\
\text { miento (7 elonga- } \\
\text { ciones, } 7 \text { veces, repeti- } \\
\text { dos } 7 \text { veces cada día). } \\
\text { Ejercicios de deglución } \\
\text { fueron completados con } \\
\text { una frecuencia de } 2 \text { veces } \\
\text { al día. }\end{array}$ & $\begin{array}{l}\text {-Mayor apertura oral ( } 2 \text { años post in- } \\
\text { tervención). } \\
\text {-Función Oromotora (a los } 6 \text { meses } \\
\text { de intervención). } \\
\text {-Eficiencia deglutoria (proporción de } \\
\text { bolo deglutido, } 3 \text { meses post inter- } \\
\text { vención). } \\
\text {-Disminución del tiempo de tránsito } \\
\text { del bolo alimenticio. } \\
\text {-Menor incidencia de alteraciones de } \\
\text { fase faríngea. } \\
\text { Calidad de Vida: } \\
\text {-Mayor proporción de usuarios que } \\
\text { se alimentan en público. }\end{array}$ \\
\hline
\end{tabular}

Reps. $=$ Repeticiones. RQT $=$ Radio-quimioterapia. HADS = Escala de Ansiedad y depresión hospitalaria. $\mathbf{H R Q O L}=$ Calidad de vida relacionada con la salud. FOIS= Escala de Ingesta Oral Funcional. VFC= Videofluoroscopia. MASA= Mann assessment swallowing ability 
se aplicaron.

\section{Enfoque Terapéutico}

El principio para mantener o mejorar la funcionalidad de las estructuras deglutorias en pacientes sometidos a RT, fundado en la neuroplasticidad, es "úselo o piérdalo", teniendo como base la especificidad, intensidad y número de repeticiones del ejercicio o maniobra terapéutica a utilizar. Es recomendable que la terapia profiláctica en pacientes con CCC sea planificada y dirigida por un fonoaudiólogo. El tratamiento en general tiene un enfoque proactivo, en queel paciente debe ejercitar y utilizar ampliamente los mecanismos deglutorios ${ }^{21}$. En ese sentido, la premisa central de la terapia proactiva es la de mitigar el desgaste orofaríngeo estructural, muscular y el remodelamiento que ocurre incluso después de breves intervalos de desuso ${ }^{32}$, permitiendo mejorar también la calidad de vida del paciente en cuanto a su alimentación ${ }^{29}$. En las investigaciones revisadas, la terapia profiláctica es ejecutada hasta 2 semanas antes del inicio de la RT o QR ${ }^{33}$, la cual debe continuar durante el tratamiento médico, teniendo una duración de 6 semanas $^{27}$ hasta 3 meses posterior al fin de la $\mathrm{RT}^{34,35}$. Además, se realizan generalmente 10 repeticiones de los ejercicios o maniobras, 2-3 veces al día los 7 días de la semana $33,35,36$.

\section{Ejercicios Deglutorios}

Estos se pueden dividir en directos, cuando la ingesta o uso de alimento está involucrado en el ejercicio, e indirectos, cuando los ejercicios para fortalecer musculatura no consideran ingesta ${ }^{8}$. El abordaje terapéutico, directo o indirecto (ya sean con o sin alimentos dentro de la cavidad oral), buscará optimizar o modificar la función deglutoria $y$, requieren de un entrenamiento activo y constante para lograr resultados. El trabajo mediante las técnicas indirectas busca mejorar el tono, la sensibilidad, la velocidad y el rango de movimiento de las estructuras orales y faríngeas que participan en el manejo del bolo alimenticio $^{37}$.

\section{a) Directos:}

Maniobra de Mendelsohn: Al deglutir, el usuario debe prestar atención al movimiento de ascenso laríngeo (como referencia puede tomar la escotadura tiroidea). En posteriores degluciones debe intentar mantener la elevación por varios segundos,contrayendo la musculatura antes de liberar ${ }^{29}$. Esta maniobra tiene como meta aumentar la extensión y la duración de la elevación laríngea y prolongar el tiempo de apertura cricofaríngea ${ }^{8}$.

Deglución con esfuerzo: Consiste en realizar una contracción muscular total exagerada mientras se deglute ${ }^{29}$. Busca optimizar la efectividad de la respuesta motora orofaríngea con la estimulación de la contracción isométrica de los músculos del piso de la boca, suprahioídeos e infrahioídeos y una contracción isotónica forzada de la musculatura faríngea ${ }^{38}$.

Deglución Supraglótica: El paciente debe respirar profundamente, contener la respiración mientras traga y luego exhalar con fuerza. Pretende proporcionar una protección volitiva de la vía aérea ${ }^{8}$.

Deglución super-supraglótica: El paciente debe inhalar conteniendo la respiración con esfuerzo, ejerciendo presión constante. Mientras se deglute, se debe continuar manteniendo la respiración y presionando, al finalizar se debe toser $^{29}$. Facilita el cierre de la entrada de la vía aérea antes y durante la deglución, aumenta el movimiento de la base de la lengua al igual que la velocidad y la extensión de la elevación laríngea ${ }^{8}$.

b) Indirectos:

Maniobra de Masako: Suavemente sostenga su lengua entre sus dientes frontales y trague su saliva manteniendo la posición lingual ${ }^{29}$. Propone fortalecer la musculatura glosofaríngea responsable de la retracción de la base de la lengua y mejorar la medialización de los constrictores faríngeos ${ }^{8}$.

Ejercicios mandibulares y de apertura oral: Tienen como objetivo mantener o mejorar los movimientos mandibulares ejercitando la musculatura deglutoria ${ }^{8}$, destacando en la revisión ejercicios de apertura y movimiento circular de la mandíbula ${ }^{36}$, movimiento mandibular contra resistencia y ejercicios pasivos de apertura oral lenta, con aparato Therabite ${ }^{33,35}$.

Ejercicios fortalecimiento-estiramiento lingual: Tienen como objetivo mejorar elrango de movimiento y fuerza de la musculatura lingual $^{8}$, dentro de estos se encuentran ejercicios de retracción lingual, en el que se debe retraer la lengua hacia la parte posterior de la boca y sostener ${ }^{29}$.

Ejercicios de Shaker: Fueron diseñados para incrementar la fuerza de la musculatura suprahioidea y aumentar el rango de apertura del esfínter esofágico superior (EES). Para este ejercicio, se instruye a los pacientes para que se 
acuesten boca arriba y levanten la cabeza para poder ver los dedos de los pies, con los hombros en el suelo. La ejecución de movimientos puede ser isométrica o isotónica ${ }^{8,36}$.

\section{DISCUSIÓN}

Las maniobras y ejercicios previamente mencionados, son los que la literatura, proveniente de ensayos clínicos aleatorizados, ha reportado como útiles en la mejoría de variables como la función deglutoria, apertura oral, severidad de la disfagia o calidad de vida, entre $\operatorname{otros}^{27,29,33,35,36,38}$. Es importante señalar que, tanto para el caso de las maniobras como los ejercicios, su selección e implementación se encuentran dirigidos con una finalidad preventiva, es decir, maximizar o mantener la funcionalidad durante el transcurso de los procedimientos de quimio y radioterapia. En ese sentido, el carácter de intensidad y duración de cada ejercicio se ve justificado por la magnitud de los correlatos anátomo-fisiológicos, como consecuencia de la terapia para el $\mathrm{CCC}^{21}$. Sin perjuicio de lo anterior, el objetivo del presente trabajo es proveer al especialista información basada en evidencia para mejorar la toma de decisiones clínicas, y dentro de ese contexto, no se debe dejar de lado la variabilidad de cada caso y, los diferentes factores que influencian en la severidad o pronóstico de un cuadro disfágico, por lo que la sugerencia además es realizar un proceso de evaluación meticuloso y reflexivo sobre cada caso particular, integrando además las preferencias de los usuarios. Para tal fin, la valoración instrumental provee de información valiosa sobre el funcionamiento de estructuras pre y post intervención ${ }^{7}$, tanto fonoaudiológica como para la condición de base.

De gran relevancia es establecer cuánto tiempo previo a la RT o QR debe iniciar la terapia profiláctica para alcanzar las metas deseadas, si bien la literatura revisada reporta un comienzo de 2 semanas previas ${ }^{30,33}$, no se aprecia un consenso absoluto o no es reportado en la totalidad de ensayos clínicos revisados. Otro elemento clave para garantizar la utilidad y efectividad de los recursos terapéuticos escogidos, especialmente cuando son procesos que se proyectará a largo plazo, sin duda lo representa la adherencia y cumplimiento al tratamiento, la cual en términos generales se reporta como alta, pero sólo hasta el momento en el que se aprecian los efectos del tratamiento para el CCC, donde desciende21, 36,39,40. En estos casos particulares, cobra más relevancia producto de la condición futura del individuo como consecuencia de la quimio-radioterapia, lo que se proyecta como un descenso lógico, tanto de la función deglutoria como de la mantención de los patrones de ejercicios. Sin embargo, resulta un desafío para los terapeutas el encontrar formas o mecanismos para poder mantener la frecuencia de la ejercitación, de manera de poder obtener cambios en la función deglutoria y la mantención de la alimentación oral, que es el objetivo a largo plazo de este tipo de programas.

Si bien en esta revisión no fueron considerados aquellos ejercicios o métodos que no han reportado efectividad en estudios experimentales, es importante señalar el caso del Entrenamiento de Fortalecimiento de la Musculatura Espiratoria (EMST, por su sigla en inglés), el cual apunta a fortalecer las fuerzas generadoras de fuerza espiratoria subglótica, lo que favorecería la protección de la vía aérea (cierre y producción de tos más eficiente), favoreciendo la eliminación de residuos en vía aérea, lo cual es considerado como prometedor en su aplicación a este tipo de usuarios, incluidos aquellos con disfagias crónicas ${ }^{14}$, pero requiere de evidencia más consistente para poder ser reafirmado.

Adicionalmente, se encuentra el caso especial de la Electroestimulación (E-stim), que ha demostrado su utilidad conjunta a maniobras o ejercicios deglutorios en usuarios con alteraciones de índole neurogénica ${ }^{41}$, sin embargo ensayos clínicos que buscaron determinar la efectividad en usuarios con CCC, demostraron que no es aconsejable su uso en ellos ${ }^{39,42}$.

\section{CONCLUSIÓN}

El presente trabajo entrega algunas nociones acerca del enfoque y recursos terapéuticos basados en evidencia para el abordaje del CCC. La selección o utilización de dichas maniobras o ejercicios deben realizarse bajo un proceso de evaluación exhaustivo y que dé cuenta de las necesidades del usuario y el impacto sobre la función deglutoria.

Finalmente, y sin perjuicio de la utilidad clínica y evidencia provista, resulta importante señalar la necesidad de mayores estudios que den más sustento a los programas terapéuticos de 
índole profiláctica, permitiendo al clínico orientar de mejor manera la toma de decisiones y pulir sus prácticas, con el objetivo de mantener o mejorar la condición deglutoria y de alimentación de sus usuarios, por lo que se insta a profundizar en dicha línea de investigación.

\section{REFERENCIAS}

1. Jemal A, Bray F, Center MM, Ferlay J, Ward E, Forman D. Global CancerStatistics.CA A Cancer J Clin [Internet]. 2011;61:69-90. Available from: https://dx.doi.org/10.3322/canjclin.49.1.33.

2. Bhatia A, Burtness B. Human papillomavirusassociated oropharyngeal cancer: Defining risk groups and clinical trials. J Clin Oncol. 2015;33(29):3243-50. Disponible en: DOI: 10.1200/ JCO.2015.61.2358

3. Thankappan K, lyer S, Menon JR. Dysphagia Management in Head and Neck Cancers. A Manual and Atlas. Kochi, India: Springer; 2018. Disponible en: https://www.springer.com/gp/ book/9789811082818

4. Platteaux N, Dirix P, Dejaeger E, Nuyts S. Dysphagia in head and neck cancer patients treated with chemoradiotherapy. Dysphagia. 2010;25(2):13952. Disponble en: https://www.researchgate.net/ publication/26771081_Dysphagia_in_Head_ and_Neck_Cancer_Patients_Treated_with_ Chemoradiotherapy

5. Pfister DG, Ang KK, Brizel DM, Burtness BA, Cmelak AJ, Colevas $A D$, et al. Head and Neck Cancers, Version 2.2014. Clinical Practice Guidelines in Oncology. JNCCN J Natl Compr Cancer Netw. 2014;12(10):1454-87.

6. Blanchard P, Bourhis J, Lacas B, Posner MR, Vermorken JB, Hernandez JJC, et al. Taxanecisplatin-fluorouracil as induction chemotherapy in locally advanced head and neck cancers: An individual patient data meta-analysis of the meta-analysis of chemotherapy in head and neck cancer group. J Clin Oncol. 2013;31(23):2854-60. Disponible en: https://www.ncbi.nlm.nih.gov/pmc/ articles/PMC6357133/

7. Denaro N, Merlano MC, Russi EG. Dysphagia in head and neck cancer patients: Pretreatment evaluation, predictive factors, and assessment during radio-chemotherapy, recommendations. Clin Exp Otorhinolaryngol. 2013:6(3):117-26. Disponible en: https://www.ncbi.nlm.nih.gov/pmc/ articles/PMC3781223/

8. Schindler A, Denaro N, Russi EG, Pizzorni N, Bossi P, Merlotti A, et al. Dysphagia in head and neck cancer patients treated with radiotherapy and systemic therapies: Literature review and consensus. Crit Rev Oncol Hematol [Internet]. 2015;96(2):372-84. Available from: http://dx.doi. org/10.1016/j.critrevonc.2015.06.005.

9. Machtay M, Moughan J, Trotti A, Garden AS, Weber RS, Cooper JS, et al. Factors associated with severe late toxicity after concurrent chemoradiation for locally advanced head and neck cancer: An RTOG analysis. J Clin Oncol. 2008;26(21):3582-9. Disponible en: DOI: 10.1200/JCO.2007.14.8841

10. Agarwal J, Dutta D, Palwe V, Gupta T, Laskar SG, Budrukkar A, et al. Prospective subjective evaluation of swallowing function and dietary pattern in head and neck cancers treated with concomitant chemo-radiation. J Cancer Res Ther. 2010;6(1):15-21. Disponible en: DOI: 10.4103/09731482.63563

11. Agarwal J, Palwe V, Dutta D, Gupta T, Laskar SG, Budrukkar A, et al. Objective assessment of swallowing function after definitive concurrent (Chemo)radiotherapy in patients with head and neck cancer. Dysphagia. 2011;26(4):399-406. Disponible en: DOI: 10.1007/s00455-011-9326-4

12. Clark BC. In vivo alterations in skeletal muscle form and function after disuse atrophy. Med Sci Sports Exerc. 2009;41(10):1869-75. Disponible en: DOI: 10.1249/MSS.0b013e3181a645a6

13. Franzmann EJ, Lundy DS, Abitol AA, Goodwin Wj. Complete hypopharyngeal obstruction by mucosal adhesions: a complication of intensive chemoradiation for advanced head and neck cancer. Head Neck. 2006;28(8):663-70. Disponible en: https://www.thelancet.com/ journals/lanonc/article/PIIS1470-2045(13)70160-8/ fulltext?code=lancet-site

14. Hutcheson KA, Barrow MP, Warneke CL, Wang Y, Eapen G, Lai SY, et al. Cough strength and expiratory force in aspirating and nonaspiratingpostradiation head and neck cancer survivors. Laryngoscope. 2018;128(7):1615-21. Disponible en: DOI: 10.1002/ lary.26986

15. Eisbruch $A$, Lyden $T$, Bradford $C R$, Dawson LA,

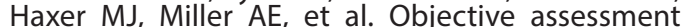
of swallowing dysfunction and aspiration after radiation concurrent with chemotherapy for headand-neck cancer. Int J Radiat Oncol Biol Phys. 2002;53(1):23-8. Diponible en: DOI: 10.1016/s03603016(02)02712-8

16. Kotz T, Costello R, Li Y, Posner MR. Swallowing dysfunction after chemoradiation for advanced squamous cell carcinoma of the head and neck. Head Neck. 2004;26(4):365-72. Disponible en: DOI: $10.1002 /$ hed.10385

17. Bernier J. Head and Neck Cancer. Multimodality Management. 2nd ed. Genolier, Switzerland: Springer; 2016.

18. Epstein JB, Chin EA, Jacobson JJ, Rishiraj B, Le $\mathrm{N}$. The relationships among fluoride, cariogenic oral flora, and salivary flow rate during radiation therapy. Oral Surg Oral Med Oral Pathol Oral Radiol Endod. 1998;86(3):286-92. Disponible en: DOl: 10.1016/s1079-2104(98)90173-1

19. Hawkins PG, Kadam AS, Jackson WC, Eisbruch A. Organ-Sparing in Radiotherapy for Head-and-Neck Cancer: Improving Quality of Life. Semin Radiat Oncol [Internet]. 2017;28(1):46-52. Available from: http://dx.doi.org/10.1016/j.semradonc.2017.08.002.

20. Ekberg O, Hamdy S, Woisard V, Wuttge-Hannig A, Ortega P. Social and psychological burden of dysphagia: Its impact on diagnosis and treatment. 
Dysphagia [Internet]. 2002;17(2):139-46. Available from: https://dx.doi.org/10.1007/s00455-001-0113-5.

21. Hutcheson KA, Bhayani MK, Beadle BM, Gold KA, Shinn EH, Lai SY, et al. Use it or lose it: Eat and exercise during radiotherapy or chemoradiotherapy for pharyngeal cancers. JAMA Otolaryngol - Head Neck Surg. 2013;139(11):1127-34. Disponible en: https:// amanetwork.com/journals/jamaotolaryngology/ fullarticle/1741150

22. Lazarus C, Logemann JA, Gibbons P. Effects of maneuvers on swallowing function in a dysphagic oral cancer patient. Head Neck. 1993;15(5):419-24. Disponible en: DOI: 10.1002/hed.2880150509

23. Lazarus C, Logemann JA, Song CW, Rademaker AW, Kahrilas PJ. Effects of voluntary maneuvers on tongue base function for swallowing. Folia Phoniatr Logop. 2002;54(4):171-6. Disponible en: DOl: $10.1159 / 000063192$

24. Logemann JA, Pauloski BR, Rademaker AW, Colangelo LA. Super-supraglottic swallow in irradiated head and neck cancer patients. Head Neck. 1997;19(6):535-40. Disponible en: DOI: 10.1002/(sici)1097-0347(199709)19:6<535::aidhed11>3.0.co:2-4

25. Rosenthal DI, Lewin JS, Eisbruch A. Prevention and treatment of dysphagia and aspiration after chemoradiation for head and neck cancer. J Clin Oncol. 2006;24(17):2636-43. Disponible en: DOl: 10.1200/JCO.2006.06.0079

26. Pauloski BR. Rehabilitation of Dysphagia Following Head and Neck Cancer. Phys Med Rehabi Clin N Am [Internet]. 2008;19(4):889-928. Available from: http://dx.doi.org/10.1016/j.pmr.2008.05.010.

27. Carnaby-Mann G, Crary MA, Schmalfuss I, Amdur R. "Pharyngocise": Randomized controlled trial of preventative exercises to maintain muscle structure and swallowing function during head-and-neck chemoradiotherapy. Int J Radiat Oncol BiolPhys [Internet]. 2012;83(1):210-9. Available from: http:// dx.doi.org/10.1016/j.ijrobp.2011.06.1954.

28. Carroll WR, Locher JL, Canon $\mathrm{CL}$, Bohannon IA, McColloch NL, Magnuson JS. Pretreatment swallowing exercises improve swallow function after chemoradiation. Laryngoscope. 2008;118(1):39-43. Disponible en: https://www.ncbi.nlm.nih.gov/pmc/ articles/PMC4312659/

29. Kotz $T$, Federman AD, Kao J, Milman L, Packer S, Lopez-Prieto C, et al. Prophylactic swallowing exercises in patients with head and neck cancer undergoing chemoradiation: A randomized trial. Arch Otolaryngol - Head Neck Surg. 2012;138(4):376-82. Disponible en: DOl: 10.1001/archoto.2012.187

30. Paleri V, Roe J, Strojan P, Corry J, Grégoire V, Hamoir M, et al. Strategies to reduce long-term postchemoradiation dysphagia in patients with head and neck cancer: An evidence-based review. Head Neck. 2014;36(3):1-13. Disponible en: DOI $10.1002 /$ hed.23251

31. Montero I, León OG. A guide for naming research studies in Psychology. Int J Clin Hea Psychol. 2007;7(3):847-62. Disponible en: https:// www.redalyc.org/pdf/337/33770318.pdf

32. Kleim JA, Jones TA. Principles of ExperienceDependent Neural Plasticity: Implications for Rehabilitation After Brain Damage. J Speech
Lang Hear Res [Internet]. 2008:51(1):225-39. Available from: https://dx.doi.org/ 10.1044/10924388(2008/018)

33. Van Der Molen L, Van Rossum MA, Rasch CRN, Smeele LE, Hilgers FJM. Two-year results of a prospective preventive swallowing rehabilitation trial in patients treated with chemoradiation for advanced head and neck cancer. Eur Arch Oto-Rhino-Laryngology. 2014;271(5):1257-70. Disponible en: DOl: 10.1007/s00405-013-2640-8

34. Ahlberg A, Engström T, Nikolaidis P, Gunnarsson $\mathrm{K}$, Johansson $\mathrm{H}$, Sharp $\mathrm{L}$, et al. Early self-care rehabilitation of head and neck cancer patients. Acta Otolaryngol. 2011;131(5):552-61. Disponible en: DOI: 10.3109/00016489.2010.532157

35. Messing BP, Ward EC, Lazarus CL, Kim M, Zhou X, Silinonte J, et al. Prophylactic Swallow Therapy for Patients with Head and Neck Cancer Undergoing Chemoradiotherapy: A Randomized Trial. Dysphagia. 2017:32(4):487-500. Disponible en: https://link.springer.com/article/10.1007/s00455017-9790-6

36. Mortensen HR, Jensen $\mathrm{K}$, Aksglæde $\mathrm{K}$ Lambertsen K, Eriksen E, Grau C. Prophylactic Swallowing Exercises in Head and Neck Cancer Radiotherapy. Dysphagia [Internet]. 2015;30(3):30414. Available from: http://dx.doi.org/10.1007/ s00455-015-9600-y.

37. Logemann JA. Dysphagia: Evaluation and Treatment. Folia Phoniatr Logop. 1995:47(1):140-64. Disponible en: DOI: 10.1159/000266348

38. Van Der Molen L, Van Rossum MA, Burkhead LM, Smeele LE, Rasch CRN, Hilgers FJM. A randomized preventive rehabilitation trial in advanced head and neck cancer patients treated with chemoradiotherapy: Feasibility, compliance, and short-term effects. Dysphagia. 2011;26(2):155-70. Disponible en: DOI: 10.1007/s00455-010-9288-y

39. Krisciunas GP, Mcculloch TM, Lazarus $C L$, Sinai M, Israel B, Pauloski BR, et al. Impact of Compliance on Dysphagia Rehabilitation in Head and Neck Cancer Patients- Results from a Multi-center Clinical Trial. Dysphagia. 2017;32(2):327-36. Disponible en: DOI: 10.1007/s00455-016-9760-4

40. Cnossen IC, van Uden-Kraan CF, Witte BI, Aalders YJ, de Goede CJT, de Bree R, et al. Prophylactic exercises among head and neck cancer patients during and after swallowing sparing intensity modulated radiation: adherence and exercise performance levels of a 12-week guided homebased program. Eur Arch Oto-Rhino-Laryngology. 2017;274(2):1129-38. Disponible en: https://research. vumc.nl/en/publications/prophylactic-exercisesamong-head-and-neck-cancer-patients-during

41. Chen YW, Chang KH, Chen HC, Liang WM, Wang $\mathrm{YH}$, Lin YN. The effects of surface neuromuscular electrical stimulation on post-stroke dysphagia: A systemic review and meta-analysis. Clin Rehabil. 2016;30(1):24-35. Disponible en: DOI: $10.1177 / 0269215515571681$

42. Langmore SE, McCulloch TM, Krisciunas GP Lazarus CL, Van-Daele D, Pauloski BR, et al. Efficacy of electrical stimulation and exercise for dysphagia in patients with head and neck cancer: A randomized clinical trial. Head Neck. 2016;38(1):1221-31. Disponible en: DOI: 10.1002/hed.24197 